\title{
The nature of flight
}

\author{
The molecules and mechanics of flight in animals
}

S ince the dawn of civilization, humans have envied their feathery companions for their ability to leap from the earth, escape gravity - and all earthly problems-and ascend to the skies. Of course, humans eventually made it into the skies too, albeit after a few millennia and rather inelegantly with the help of technology. Even so, we can now fly around the Earth in a matter of hours or zoom about in a fighter plane at supersonic speeds. Yet, flying has never lost its fascination for humans, and many scientists continue to study how various species have mastered the physical and metabolic challenges of flight. Many of the basic questions regarding aerodynamics and the physical mechanics of flightincluding the fundamental one of how the bumblebee manages to stay in the airhave largely been resolved. Now, the spotlight has moved to focus on the underlying metabolic and molecular mechanisms that enable animals to fly.

The ability to fly appears to have evolved separately at least four times: in birds, bats, insects and pterosaurs. Although pterosaurs are extinct, the other three provide unique opportunities to study the aerodynamic and molecular features of animal flight. The evolution of flight in both vertebrates and invertebrates has led to a wide variety of adaptations to exploit unique physiological advantages and to overcome specific deficiencies. For example, size matters and, owing to various mathematical laws, smaller animals have to flap their wings faster to stay in the air. Furthermore, air resistance is a more significant problem for smaller animals, which means that there is an effective minimum size for flight. Yet other factors mean that flying animals also cannot be too large; oxygen and sugars have to travel further around the body, and the power required for take off is proportionately greater-as can be seen by watching how large birds such as swans or albatrosses struggle to get airborne. Recent research into Argentavis magnificens - the largest bird ever known to have flown-demonstrated that its $7 \mathrm{~m}$ wingspan was so great that it simply could not have taken off from the ground. The mathematical model, created by Sankar Chatterjee and co-workers, and based on fossil bones of the bird and on computer flight models, demonstrated that Argentavis must have launched from high places, using thermals and updrafts to gain height before gliding for up to 200 miles at a time (Chatterjee et al, 2007).

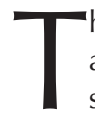
he phenomenon of hovering flight is a good starting point from which to study how animals have adapted their bodies to be able to fly. Hovering is particularly energy-demanding and has evolved separately in birds, insects and mammals that feed on plant nectar. Although hovering does not consume energy quite as quickly as chasing prey or evading predators, it must be sustained for longer periods and therefore requires huge amounts of fuel and the ability to metabolize it efficiently. Hummingbirds are the champions of hovering and consume around five times their body weight in fuel per day. Their hearts beat at up to 1,200 beats per minute during flight and are exceptionally large in proportion to the size of the birds (Altshuler \& Dudley, 2002).

Hummingbirds are also one of the largest and most successful avian groups with 320 known species occupying a wide variety of ecological niches in the Americas. Some have mastered hovering at altitudes of up to

analysis ancher . 
their wings alone. Feathers obviously provide additional energy savings, perhaps by exploiting local turbulences to reduce drag and increase lift (Altshuler et al, 2004).

\section{The evolution of flight in both vertebrates and invertebrates has led to a wide variety of adaptations to exploit unique physiological advantages and to overcome specific deficiencies}

In contrast to birds, some of which hover easily or travel huge distances, bats-the other group of flying vertebrates-seem to be rather second-rate flyers. There are a few 'flower bats' that are able to hover, but only close to sea level. "Although bats can hover transiently, perhaps up to a minute, they cannot hover for as long as hummingbirds or insects," said Douglas Altshuler, Assistant Professor of Biology and an animal flight specialist at the University of California (Riverside, CA, USA). Furthermore, although bats travel significant distances in search of food, their range tends to be around $800 \mathrm{~km}$ at most, while birds migrating between the Arctic and Antarctic cover more than $15,000 \mathrm{~km}$. Avian adaptations have allowed birds to conquer almost the whole world, both in terms of latitude and altitude, and also to perfect hovering to an extent that bats have never achieved.

But it would be a mistake to consider bats inferior to birds. They have fundamentally different wings and, although birds win in terms of efficiency and stamina, bats have the edge in aerobatic ability. Bat wings are extensions of mammalian arms and are formed by membranes with double layers of skin that join the bones of the fingers. The membrane contains a combination of muscle and elastic fibres to keep it stiff but enable it to fold. Bats can use their 'fingers' to adapt the shape of the wing to perform the highly precise acrobatic movements needed to catch insects in the air.

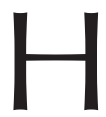

owever, despite recent research into the flight of bats and hummingbirds, arguably the most exciting insights have come from flying insects, in particular about the structure of the flight muscles and their coordination with the central nervous system. Insects evolved flight much earlier than birds or bats and exhibit some of the most profound genetic adaptations. They also evolved flight with much smaller body sizes, which brings both challenges and opportunities. It seems that insect flight has gone through two stages of evolution, with some of the most successful species, including bees and all beetles, being in the second wave (Iwamoto et al, 2006).

The crucial difference between the two modes of insect flight is the signalling between the nervous system and the wings. All vertebrates and many insects, such as butterflies, use the more 'primitive' mode of flight, whereby wing beats are controlled directly by the nervous system. This mechanism is called synchronous flight because each wing beat is generated by a single nerve impulse-in the same way that humans and all mammals manipulate their limbs. Synchronous flight works well for birds, bats and larger insects, but seems not to function for body sizes smaller than that of a bumblebee. Below this size, the frequency of wing beats required to support flight becomes unsustainable using the synchronous flight model_-it is physically impossible to trigger muscle contractions quickly enough. In synchronous flight, the nerve impulses trigger contractions by causing the sarcoplasmic reticulum to release stored calcium. When the calcium is subsequently pumped back to the sarcoplasmic reticulum, the contraction ends and the muscles relax, but there is a physical limit to how quickly the calcium can be moved. This 'speed limit' constrains the maximum wing beat frequency to around $100 \mathrm{MHz}$ in synchronous operation.

\section{...during the course of their evolution, hummingbirds were able to take several avian adaptations to their absolute limit}

One possible solution to extend this limit is to increase the volume of the sarcoplasmic reticulum so that calcium can be pumped in and out in parallel-in addition to a denser mitochondrial population in the muscle cells to produce energy more quickly. This solution has been adopted by the few vertebrates that have succeeded in achieving twitches faster than $100 \mathrm{MHz}$ in non-flight muscles, such as the rattlesnake and the sound-producing toadfish. However, this is not sustainable for long periods because it consumes too much energy. According to Hiroyuki Iwamoto, who studies insect flight muscles at the Japan Synchrotron Radiation Research Institute in Hyogo, Japan, "for continually flying insects, it is not wise to consume a lot of energy for sequestering calcium."

Some insects have therefore evolved a completely different mechanism to control wing beats. This mechanism is called asynchronous flight because the nerve impulses are no longer associated directly with the wing beats and, in effect, the nervous system of the insect communicates with a 'flight engine' to which it delegates the task of controlling the flight muscles. This system involves subordinate muscles that activate the primary flight muscle by stretching it through a mechanism that is not yet fully understood. "These insects control their flight by means of a set of accessory 'steering muscles' rather than the major flight muscles themselves," Iwamoto said. Asynchronous flight makes hovering and other aerodynamic feats, such as flying backwards, easier to achieve and sustain. It has also allowed insects to reduce their size and to exploit local and transient pressure changes in the air to increase uplift. The physics are complex but, in essence, the wings are able to create pressure gradients that give added uplift to the insect (Dickinson et al, 1998).

A Ithough the exact mechanisms of asynchronous flight are not yet fully understood, one important discovery was made recently. Micro-X-ray analysis of frozen flight muscle showed that the myofibrils are extraordinarily symmetrical, with each successive multi-protein lattice-called a sarcomere-arranged in precise alignment. According to Iwamoto, who first discovered this in the bumblebee, the maximum misalignment between successive sarcomeres is at most $0.003^{\circ}-$ these myofibrils are in effect giant protein crystals (Iwamoto et al, 2002). "This regularity is amazing, considering that each myofilament is only $1-2 \mu \mathrm{m}$ long and the myofilaments are disrupted at every Z-line that separates two neighbouring sarcomeres," commented Iwamoto. He and his team went on to show that all asynchronous flight muscles exhibit this symmetry (Iwamoto et al, 2006).

The crystalline structure of asynchronous flight muscles has probably evolved independently several times. The X-ray diffraction patterns revealed similar regular hexagonal arrays in bees (Hymenoptera), 
flies (Diptera), beetles (Coleoptera) and true bugs (Heteroptera); as the last common ancestor of these groups probably flew synchronously, each group is likely to have evolved asynchronous flight independently. Furthermore, even large beetles that do not necessarily require asynchronous flight-because they beat their wings slowly-maintain this ordered myofibril structure. Iwamoto suggested that their smaller ancestors probably evolved asynchronous flight and that the larger beetles retained it even when it was no longer completely necessary.

In fact, asynchronous flight can be a disadvantage for larger insects with relatively slow wing beats. Large longhorn beetles, for example, which use asynchronous flight muscles like all beetles, are far clumsier in the air than other big insects with synchronous flight. Iwamoto commented that both modes have advantages and disadvantages, and that asynchronous flight is not intrinsically superior. At a reduced frequency of wing beats it becomes an advantage to control flight speed and direction by varying each flap: "Insects with synchronous flight muscles, such as butterflies, can swing their wings in various ways, and some can keep their wings horizontal and glide for a long distance, probably without spending much energy," he said.

\section{...although birds win in terms of efficiency and stamina, bats have the edge in aerobatic ability}

This raises the question of why the crystalline structure is apparently crucial to asynchronous flight? One clue comes from those insects that have not evolved it, but still need to hover with relatively fast wing beats to feed on nectar. The hummingbird hawk moth is such an insect: it beats its wings at up to $85 \mathrm{MHz}$ - which is approaching the maximum frequency for synchronous flight. Iwamoto examined the myofibril diffraction pattern of the hawk moth and found that they exhibited some regularity in the orientation of their lattice planes, but not as much as in the muscles of asynchronous insects (Iwamoto et al, 2006). "I speculate that increasing demands for skilful flight may drive the myofibrillar structure toward the giant single-crystal type," he said. The evolution of partial crystalline order in the myofibrils seems to be a necessary precursor to asynchronous flight, which allows increasing wing beat frequencies towards the limit for synchronous operation. A second clue is that, even in insects that fly asynchronously, the crystalline myofibril structure is confined to just the flight muscles. But again, this does not fully explain why the crystalline structure is so important, with the best guess being that it provides the strength or stiffness required for fast flapping and enables force to be transmitted more efficiently along the muscle fibres.

$\mathrm{H}$ owever, significant progress has been made in elucidating some other features of insect flight muscles. The main demands of asynchronous flight are rapid activation and deactivation of the flight muscles, and the efficient delivery of energy. The rapid pumping of calcium from and to the sarcoplasmic reticulum, which normally occurs in muscle cells where the contraction is synchronized with nerve cells, is discarded in asynchronous flight. This allows more rapid contractions to be controlled by resonance between the wing and the thorax of the insect. It also saves most of the space occupied by the sarcoplasmic reticulum in the muscle cells of synchronous flying insects. About half the total muscle volume can then be dedicated to energy-producing mitochondria and the other half to the myofibrils that do the work (Bullard et al, 2005).

Another unique adaptation for asynchronous flight is a protein called flightin, which gives the myofibrils the stiffness required for rapid wing beats without losing elasticity. It is found only in asynchronous flight muscles, in addition to kettin and projectin-proteins that are present in all flight muscles of insects (Bullard et al, 2005). Experiments have shown that insects with a null mutation in the flightin gene are much poorer flyers (Barton et al, 2005). Furthermore, mechanical analysis of myofibrils that lacked flightin showed that they had lost the required stiffness to sustain rapid wing beats. In any case, there are significant differences between insect species and the ways in which they fly; it is not a case of 'one size fits all'. Recent work has shown that evolutionary adjustments in the elastic properties of insect wings have come about through small changes in various constituent proteins, perhaps through the selection of particular alleles within a population to produce different isoforms (Bullard et al, 2005).

But some insects have more specialized flight requirements than others. Although there is a general relationship between insect size and wing beat frequency, the honeybee has a much faster wing beat than would be expected on this basis alone. This mystery was cleared up in 2005, when Douglas Altshuler and colleagues at the California Institute of Technology (Pasadena, CA, USA) showed that honeybees use a wing stroke of shorter amplitude but higher frequency than other asynchronous insects of the same size (Altshuler et al, 2005). This adaptation generates the extra power that they need to carry heavy loads of nectar or larvae.

Although research into the physics, metabolism and molecular adaptations of flight is answering many questions, it is also creating new ones. Given the unique adaptations some species have evolved to become successful flyers, it is fascinating to discover the ways in which various species have pushed their physical and metabolic abilities to the very limits. Although humans remain with solid feet of clay, the age-old dream of flight is still an irresistible fascination for those who want to understand how it is achieved.

\section{REFERENCES}

Altshuler DL, Dudley R (2002) The ecological and evolutionary interface of hummingbird flight physiology. J Exp Biol 205: 2325-2336

Altshuler DL, Dudley R, Ellington CP (2004) Aerodynamic forces of revolving hummingbird wings and wing models. J Zoo/ 264: 327-332

Altshuler DL, Dickson WB, Vance JT, Roberts SP, Dickinson MH (2005) Short-amplitude high-frequency wing strokes determine the aerodynamics of honeybee flight. Proc Natl Acad Sci USA 102: 18213-18218

Barton B, Ayer G, Heymann N, Maughan DW, Lehmann FO, Vigoreaux JO (2005) Flight muscle properties and aerodynamic performance of Drosophila expressing a flightin transgene. J Exp Biol 208: 549-560

Bullard B, Burkart C, Labeit S, Leonard K (2005) The function of elastic proteins in the oscillatory contraction of insect flight muscle. I Muscle Res Cell Motil 26: 479-485

Chatterjee S, Templin RJ, Campbell KE Jr (2007) The aerodynamics of Argentavis, the world's largest flying bird from the Miocene of Argentina. Proc Natl Acad Sci USA [doi:10.1073/pnas.0702040104]

Dickinson MH, Lehmann FO, Chan WP (1998) The control of mechanical power in insect flight. Am Zool 38: 718-728

Iwamoto H, Nishikawa Y, Wakayama J, Fujisawa T (2002) Direct X-ray observation of a single hexagonal myofilament lattice in native myofibrils of striated muscle. Biophys / 83: 1074-1081

Iwamoto H, Inoue K, Yagi N (2006) Evolution of long-range myofibrillar crystallinity in insect flight muscle as examined by $\mathrm{X}$-ray cryomicrodiffraction. Proc Biol Sci 273: 677-685

\section{Philip Hunter}

doi:10.1038/sj.embor.7401050 